Review Article

\title{
Effect of Epidural Dexmedetomidine as an Adjuvant to Local Anesthetics for Labor Analgesia: A Meta-Analysis of Randomized Controlled Trials
}

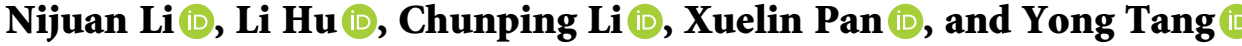 \\ Department of Anesthesia, Sichuan Jinxin Women and Children Hospital, Chengdu 610000, China \\ Correspondence should be addressed to Yong Tang; 1960544843@qq.com
}

Received 27 May 2021; Accepted 16 October 2021; Published 28 October 2021

Academic Editor: Rodolfo Parreira

Copyright (c) 2021 Nijuan Li et al. This is an open access article distributed under the Creative Commons Attribution License, which permits unrestricted use, distribution, and reproduction in any medium, provided the original work is properly cited.

\begin{abstract}
Background. This study aims to determine the analgesic effect and safety of dexmedetomidine as an adjuvant to epidural local anesthetics during labor. Methods. Randomized controlled trials comparing epidural blocks with or without dexmedetomidine for labor analgesia were comprehensively searched. Review manager 5.4 was used to analyze the extracted data. Results. Compared with placebo and opioids, dexmedetomidine relieved labor pain of $15 \mathrm{~min}(P=0.002), 30 \mathrm{~min}(P=0.01)$, and $120 \mathrm{~min}(P=0.02)$ after block and at the moment of fetal disengagement $(P=0.0002)$, decreased mean arterial pressure of $120 \mathrm{~min}(P=0.01)$, heart rate of $30 \mathrm{~min}(P=0.003), 60 \mathrm{~min}(P<0.00001)$, and $120 \mathrm{~min}(P<0.00001)$ after block, blood loss $(P=0.02)$, and the incidence of nausea/vomiting $(P=0.006)$, and increased the incidence of maternal bradycardia $(P=0.04)$. However, sensitivity analysis only found that the incidence of nausea/vomiting was significantly different. Compared with placebo, dexmedetomidine relieved labor pain of 30 min after block $(P<0.00001)$ and did not increase the incidences of side effects, but only two studies were enrolled. Compared with opioids, dexmedetomidine decreased the incidence of nausea/vomiting $(P=0.002)$, increased the incidence of maternal bradycardia $(P=0.04)$, and had a similar effect on labor pain relief; however, sensitivity analysis found that significant difference existed only at the incidence of nausea/vomiting. Other outcomes from meta-analysis or subgroup analysis were not different. Conclusions. Epidural dexmedetomidine has the potential to offer a better analgesic effect than placebo, similar labor pain control to opioids, and has no definite adverse effects on the parturient or fetus, but more high-quality studies are needed to confirm these conclusions.
\end{abstract}

\section{Introduction}

Labor pain causes significant suffering to parturients, both physically and mentally. Additionally, it increases the risk of childbirth $[1,2]$ and, to some extent, increases the rate of cesarean section [3]. Labor analgesia refers to the use of various methods to reduce or eliminate maternal labor pain and is increasingly applied in obstetrics [3]. The features of ideal labor analgesia include maternal and fetal safety, rapid onset, good analgesic effect, and few adverse reactions [3].

Currently, epidural analgesia with local anesthetics has been the most effective and preferred choice for labor and delivery $[4,5]$. However, it also has some disadvantages during labor, including motor blockade, maternal hypotension, longer second stage of labor, and urinary retention
[6]. Thus, a major challenge for anesthesiologists is the correct balance of local anesthetics and the management of complications. Local anesthetics combined with opioids were used to address these challenges. However, it has been shown that epidural opioids are often accompanied by adverse effects, such as respiratory depression, lethargy, pruritus, nausea, and vomiting [7].

Dexmedetomidine, a highly selective $\alpha 2$-adrenoceptor agonist, possesses anxiolytic, sedative, and analgesic properties without causing respiratory depression $[8,9]$. Moreover, dexmedetomidine combined with local anesthetics has been successfully used for epidural labor analgesia with few side effects $[10,11]$; however, no published meta-analysis has evaluated the effect of epidural dexmedetomidine on labor analgesia. To provide a high evidence level for clinical 
application, we conducted a meta-analysis comparing dexmedetomidine with placebo or opioids as an adjuvant to local anesthetics for labor analgesia with respect to analgesic effect and safety.

\section{Methods}

This meta-analysis was conducted by following recommendations of the Cochrane Handbook for Systematic Reviews of Interventions [12] and the Preferred Reporting Items for Systematic Reviews and Meta-Analyses (PRISMA) [13]. The study was registered with PROSPERO (CRD42020167287).

2.1. Search Strategy. Two authors comprehensively searched Embase (1980-2020.08), PubMed (1966-2020.08), Medline (1966-2020.08), and the Cochrane Library using keywords ("labor OR labour OR vaginal delivery OR vaginal," "analgesia OR pain," and "dexmedetomidine") without language restriction. The databases of https://clinicaltrials.gov/ and Chinese Clinical Trial Registry were also searched by using the following keywords: "labor OR labour OR vaginal delivery OR vaginal" and "dexmedetomidine." The search strategy can be found in Appendix A. The references of the identified trials and systematic reviews were also manually searched to identify any potentially relevant trials.

2.2. Inclusion Criteria. Trials were included in our metaanalysis to determine whether they met the PICOS (patients, intervention, comparator, outcome, and study design) criteria. (1) Patients: nulliparous women undergoing epidural anesthesia during labor. (2) Intervention: epidural block with epidural dexmedetomidine for labor analgesia without dosage restriction. (3) Comparator: epidural block with or without epidural opioids (morphine, fentanyl, sufentanil, and remifentanil) for labor analgesia. (4) Outcomes: visual analog scale (VAS) for pain, mode of delivery, duration of labor, blood pressure, heart rate (HR), blood loss, onset of analgesia, motor block, level of sedation, complications, fetal 1-min and 5-min Apgar scores, and umbilical artery pH and partial pressure of oxygen $\left(\mathrm{PaO}_{2}\right)$. (5) Study design: randomized controlled trials (RCTs). Trials with insufficient outcome data were excluded. Two authors independently assessed the eligibility of studies. In cases of disagreement, a consensus was reached through discussion with a third author where necessary.

2.3. Data Extraction. Two reviewers independently retrieved the relevant data from the articles using a standard data extraction form. The primary outcome was the VAS score on a $0-10$ scale during labor. If the pain scale was assessed by a $0-100$ scale, then, it was converted to a $0-10$ scale by dividing by 10 . The secondary outcomes were the mode of delivery, duration of labor, onset of analgesia, blood pressure, HR, blood loss, motor block, level of sedation, complications, 1min and 5-min Apgar scores, and umbilical artery $\mathrm{pH}$ and $\mathrm{PaO}_{2}$. Missing data were requested from study authors.
2.4. Assessment of Methodological Quality. Two authors assessed the quality of the included studies independently based on the guidelines in the Cochrane Handbook for Systematic Reviews of Interventions and a modified Jadad 7point scale. A study with a modified Jadad score $<4$ points is regarded as low-quality [14]. If the number of included RCTs was $\geq 10$, publication bias was assessed by a funnel plot [15].

2.5. Statistical Analysis. Review Manager software 5.4 was used for meta-analysis. Mean differences (MDs) with 95\% confidence intervals (CIs) were used to assess continuous outcomes. Odds ratios (ORs) with 95\% CIs were used to assess dichotomous outcomes. Statistical heterogeneity among the included studies was assessed by $P$ and $I^{2}$. The control group included placebo and opioids, so all metaanalyses were conducted by using a random-effect model. The inverse variance and Mantel-Haenszel methods were used to combine separate statistics. Sensitivity analysis was conducted by omitting one study in turn to examine the reliability and conclusiveness of the available evidence. Subgroup analysis was conducted in accordance with different controls (placebo or opioids). A $P$ value less than 0.05 was considered significant.

Additionally, trial sequential analysis (TSA) software 0.9.5.10 Beta was used to examine the reliability and conclusiveness of the available evidence according to a previous meta-analysis $[16,17]$. To calculate the required information size (RIS), all outcomes used two-sided tests with a type I error of $5 \%$ and a power of $80 \%$, continuous outcomes used an empirical mean difference, and dichotomous outcomes used a low bias-based relative risk reduction and a literaturebased incidence in controls (5\% for nausea/vomiting [18]).

\section{Results}

3.1. Search Results. Throughout the search strategy, a total of 178 studies were identified, and 155 studies were excluded by reading the title and abstract (Figure 1). Then, 11 registered clinical trials with no data available and one study [19] with no full text available were excluded. Two further articles were excluded after reading the full texts of the remaining article in detail. One article [10] compared different contents of dexmedetomidine, and the second [20] compared intravenous dexmedetomidine and remifentanil with remifentanil. Finally, nine studies $[3,11,21-27]$ with 1,403 patients were included in our meta-analysis.

The basic characteristics and interventions are summarized in Table 1. Eight RCTs assessing ropivacaine were carried out in China [3, 11, 22-27], and one assessing bupivacaine was carried out in Egypt [21]. Five studies $[3,11,22,25,27]$ compared dexmedetomidine with placebo, one [21] compared dexmedetomidine with fentanyl, and three $[23,24,26]$ compared dexmedetomidine with sufentanil. Six RCTs [3, 22-26] used $0.5 \mu \mathrm{g} / \mathrm{mL}$ dexmedetomidine, one [11] used $0.5 \mu \mathrm{g} / \mathrm{kg}$ dexmedetomidine, one [21] used $1 \mu \mathrm{g} / \mathrm{kg}$ dexmedetomidine, and one [27] used four concentrations of dexmedetomidine. Eight studies [3, 11, 21, 23-27] with Jadad scores $\geq 4$ were regarded as of high-quality. 


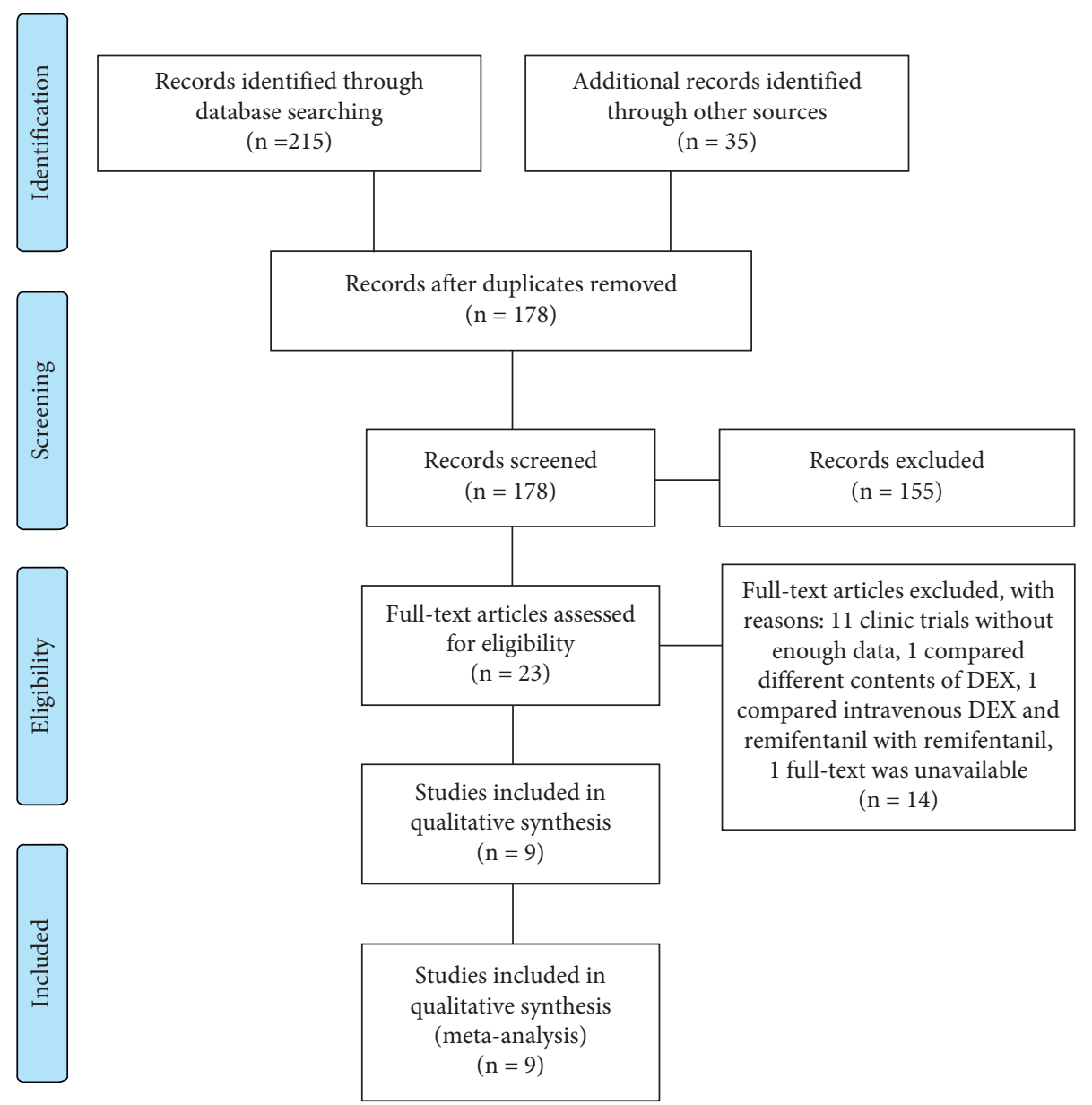

FIGURE 1: PRISMA flow chart for study selection.

3.2. Risk of Bias Assessment. The risk of bias assessments is presented in Figure 2. Six RCTs [11, 23-27] were recorded using a computer or a random number generator for randomization, and three $[23,26,27]$ reported allocation concealment via sealed envelopes. A double-blind method was carried out in six studies [21, 23-27], and the implementation of blinding of outcomes was carried out in five studies [21, 23, 25, 27]. One study [25] had a high risk of bias for incomplete outcome data, and one study [11] had a high risk of bias for selective reporting. Five studies [23-27] presented no other bias.

\subsection{Results of Meta-Analysis}

3.3.1. Primary Outcomes. Statistical differences in VAS scores (Table 2, Supplementary Figure 1) between the dexmedetomidine group and control group were detected at $15 \mathrm{~min} \quad(P=0.002), \quad 30 \mathrm{~min} \quad(P=0.01)$, and $120 \mathrm{~min}$ $(P=0.02)$ after block and at the moment of fetal disengagement $(P=0.0002)$. However, heterogeneities existed among the included studies (except VAS score before epidural block, others had $I^{2}>50 \%$, Table 2 and Supplementary Figure 1).
3.3.2. Secondary Outcomes. Statistical differences (Table 2, Supplementary Figures 2-5) between the dexmedetomidine group and control group were detected with the measurement of mean arterial pressure (MAP) of $120 \mathrm{~min}(P=0.01)$ after block, HR of $30 \mathrm{~min}(P=0.003), 60 \mathrm{~min}(P<0.00001)$, and $120 \mathrm{~min}(P<0.00001)$ after block, blood loss $(P=0.02)$, and incidences of maternal bradycardia $(P=0.04)$ and nausea/vomiting $(P=0.006)$. Other outcomes, including the duration of first and second stages, mode of delivery, onset of analgesia, MAP of $15 \mathrm{~min}, 30 \mathrm{~min}$, and $60 \mathrm{~min}$ after block and at the moment of fetal disengagement, HR of $15 \mathrm{~min}$ after block and the moment of fetal disengagement, level of motor block assessed by a modified Bromage scale, 1-min and 5-min Apgar scores, umbilical artery $\mathrm{pH}$ and $\mathrm{PaO}_{2}$, and incidences of hypotension, itching, shivering, urinary retention, and fetal HR abnormality, were not significantly different (Table 2, Supplementary Figures 2-11). Heterogeneities among studies were detected at the time of the first $\left(I^{2}=92 \%\right)$ and second $\left(I^{2}=97 \%\right)$ stages, onset of analgesia $\left(I^{2}=93 \%\right)$, MAP of $15 \mathrm{~min}\left(I^{2}=91 \%\right)$ and $30 \mathrm{~min}\left(I^{2}=52 \%\right)$ after block, and at the moment of fetal disengagement $\left(I^{2}=92 \%\right)$, HR of 15 -min after block $\left(I^{2}=93 \%\right)$ and at the moment of fetal disengagement $\left(I^{2}=96 \%\right)$, level of motor block $\left(I^{2}=95 \%\right)$, and umbilical artery $\mathrm{PaO}_{2}\left(I^{2}=96 \%\right)$. 
TABLE 1: The characteristics of included studies.

\begin{tabular}{|c|c|c|c|c|c|c|c|c|c|c|c|c|}
\hline \multirow[b]{2}{*}{$\begin{array}{l}\text { Study } \\
\text { (year) }\end{array}$} & \multirow[b]{2}{*}{ Country } & \multirow[b]{2}{*}{ Cases } & \multicolumn{6}{|c|}{$\mathrm{DEX} /$ control } & \multirow[b]{2}{*}{$\begin{array}{l}\text { Epidural } \\
\text { analgesia }\end{array}$} & \multirow[b]{2}{*}{$\begin{array}{c}\text { DEX } \\
\text { intervention }\end{array}$} & \multirow[b]{2}{*}{$\begin{array}{c}\text { Control } \\
\text { intervention }\end{array}$} & \multirow[b]{2}{*}{$\begin{array}{r}\text { Jadac } \\
\text { score }\end{array}$} \\
\hline & & & $\begin{array}{l}\text { Mean } \\
\text { age } \\
\text { (years) }\end{array}$ & $\begin{array}{l}\text { Gestational } \\
\text { week }\end{array}$ & $\begin{array}{l}\text { Weight } \\
(\mathrm{kg})\end{array}$ & $\begin{array}{l}\text { Height } \\
(\mathrm{cm})\end{array}$ & $\begin{array}{c}\text { BMI } \\
\left(\mathrm{kg} / \mathrm{m}^{2}\right)\end{array}$ & $\begin{array}{l}\text { Cervical } \\
\text { dilatation } \\
(\mathrm{cm})\end{array}$ & & & & \\
\hline $\begin{array}{l}\text { Jun } \\
\text { et al. } \\
{[3]}\end{array}$ & China & $75 / 75$ & $\begin{array}{l}25.5 / \\
26.5\end{array}$ & $37.6 / 36.9$ & $\begin{array}{l}61.4 / \\
62.8\end{array}$ & $\begin{array}{l}166.1 / \\
165.5\end{array}$ & NM & $\mathrm{NM}$ & $\begin{array}{l}\text { When the } \\
\text { cervical } \\
\text { dilation } \\
\text { reached } 3 \mathrm{~cm} \text {, } \\
\text { a catheter was } \\
\text { inserted at the } \\
\text { L3-L4 } \\
\text { interspace, } \\
3-4 \mathrm{~cm} \\
\text { cephaladly. } \\
\text { First dose: } \\
10 \mathrm{~mL} \text {, } \\
\text { background: } \\
10 \mathrm{~mL} / \mathrm{h}, \\
\text { bonus: } 5 \mathrm{~mL} \text {, } \\
\text { lockout time: } \\
20 \mathrm{~min}\end{array}$ & $\begin{array}{c}\text { PCEA: } 0.1 \% \\
\text { ropivacaine } \\
\text { and } 0.5 \mu \mathrm{g} / \\
\text { mL DEX; first } \\
\text { dose: } 10 \mathrm{ml} \text {, } \\
\text { background } \\
\text { infusion: } \\
10 \mathrm{ml} / \mathrm{h} \text {, a } \\
\text { single } \\
\text { additional } \\
\text { quantity: } \\
5 \text { ml, and lock } \\
\text { time: } 20 \text { min }\end{array}$ & $\begin{array}{c}\text { PCEA: } 0.1 \% \\
\text { ropivacaine; } \\
\text { first dose: } \\
10 \mathrm{ml} \text {, } \\
\text { background } \\
\text { infusion: } \\
10 \mathrm{ml} / \mathrm{h}, \mathrm{a} \\
\text { single } \\
\text { additional: } \\
\text { quantity } 5 \mathrm{ml} \text {, } \\
\text { and lock time: } \\
20 \mathrm{~min}\end{array}$ & 4 \\
\hline $\begin{array}{l}\text { Zhao } \\
\text { et al. } \\
{[11]}\end{array}$ & China & $40 / 40$ & $\begin{array}{l}25.9 / \\
26.2\end{array}$ & NM & $\begin{array}{l}80.7 / \\
79.3\end{array}$ & $\begin{array}{l}163.2 / \\
163.03\end{array}$ & $\begin{array}{l}30.3 / \\
29.9\end{array}$ & NM & $\begin{array}{l}\text { A catheter } \\
\text { was advanced } \\
\text { through the } \\
\text { needle } 4 \mathrm{~cm} \\
\text { into the } \\
\text { epidural space }\end{array}$ & $\begin{array}{c}0.125 \% \\
\text { ropivacaine } \\
\text { with } 0.5 \mu \mathrm{g} / \mathrm{kg} \\
\text { DEX }\end{array}$ & $\begin{array}{l}0.125 \% \\
\text { ropivacaine }\end{array}$ & 5 \\
\hline $\begin{array}{l}\text { Selim } \\
\text { et al. } \\
{[21]}\end{array}$ & Egypt & $44 / 43$ & $\begin{array}{l}25.1 / \\
24.0\end{array}$ & $38.9 / 39.2$ & $\begin{array}{l}74.7 / \\
78.1\end{array}$ & $\begin{array}{l}161 / \\
163\end{array}$ & $\mathrm{NM}$ & $5.1 / 4.9$ & $\begin{array}{c}\text { A catheter } \\
\text { was inserted } \\
\text { into the L3-L4 } \\
\text { interspace, } \\
2-3 \mathrm{~cm} \\
\text { cephaladly. } \\
\text { Initial bolus } \\
17 \mathrm{~mL} \text {, a } \\
\text { second dose } \\
\text { was injected } \\
\text { when VAS } \\
\text { was } \geq 4\end{array}$ & $\begin{array}{c}12 \mathrm{~mL} \text { of } \\
0.25 \% \\
\text { bupivacaine } \\
\text { plus } 1 \mu \mathrm{g} / \mathrm{kg} \\
\text { DEX diluted } \\
\text { in } 5 \mathrm{~mL} \text { saline }\end{array}$ & $\begin{array}{c}12 \mathrm{~mL} \text { of } \\
0.25 \% \\
\text { bupivacaine } \\
\text { plus } 1 \mu \mathrm{g} / \mathrm{kg} \\
\text { fentanyl } \\
\text { diluted in } \\
5 \mathrm{~mL} \text { saline }\end{array}$ & 5 \\
\hline $\begin{array}{l}\text { Zhang } \\
\text { and Li } \\
{[22]}\end{array}$ & China & $30 / 30$ & $\begin{array}{l}28.0 / \\
26.9\end{array}$ & $39.2 / 38.7$ & $\begin{array}{l}71.3 / \\
68.5\end{array}$ & $\begin{array}{c}159.4 / \\
160.5\end{array}$ & $\mathrm{NM}$ & $2.0 / 2.1$ & $\begin{array}{l}\text { When the } \\
\text { cervical } \\
\text { dilation } \\
\text { reached } 2 \mathrm{~cm} \text {, } \\
\text { a catheter was } \\
\text { inserted at the } \\
\text { L2-L3 } \\
\text { interspace, } \\
3-5 \mathrm{~cm} \\
\text { cephaladly. } \\
\text { First dose } \\
10 \mathrm{~mL} \text {, } \\
\text { background } \\
8 \mathrm{~mL} / \mathrm{h} \text {, bonus } \\
8 \mathrm{~mL}, \text { lockout } \\
\text { time } 15 \text { min }\end{array}$ & $\begin{array}{l}10 \mathrm{ml} \text { of } 0.1 \% \\
\text { ropivacaine } \\
\text { plus } 0.5 \mu \mathrm{g} / \\
\mathrm{mL} \mathrm{DEX}, \\
\text { background } \\
\text { infusion } 8 \mathrm{ml} / \\
\mathrm{h} \text {, a bolus of } \\
8 \mathrm{ml} \text { when } \\
\mathrm{VSA}>7, \\
\text { lockout time } \\
15 \mathrm{~min}\end{array}$ & $\begin{array}{l}10 \mathrm{ml} \text { of } 0.1 \% \\
\text { ropivacaine, } \\
\text { background } \\
\text { infusion: } \\
8 \mathrm{ml} / \mathrm{h}, \mathrm{a} \\
\text { bolus of } 8 \mathrm{ml} \\
\text { when } \\
\mathrm{VSA}>7 \text {, } \\
\text { lockout time: } \\
15 \mathrm{~min}\end{array}$ & 3 \\
\hline
\end{tabular}


TABLE 1: Continued.

\begin{tabular}{|c|c|c|c|c|c|c|c|c|c|c|c|c|}
\hline \multirow[b]{2}{*}{$\begin{array}{l}\text { Study } \\
\text { (year) }\end{array}$} & \multirow[b]{2}{*}{ Country } & \multirow[b]{2}{*}{ Cases } & \multicolumn{6}{|c|}{ DEX/control } & \multirow[b]{2}{*}{$\begin{array}{l}\text { Epidural } \\
\text { analgesia }\end{array}$} & \multirow[b]{2}{*}{$\begin{array}{c}\text { DEX } \\
\text { intervention }\end{array}$} & \multirow[b]{2}{*}{$\begin{array}{c}\text { Control } \\
\text { intervention }\end{array}$} & \multirow[b]{2}{*}{$\begin{array}{r}\text { Jadad } \\
\text { score }\end{array}$} \\
\hline & & & $\begin{array}{l}\text { Mean } \\
\text { age } \\
\text { (years) }\end{array}$ & $\begin{array}{l}\text { Gestational } \\
\text { week }\end{array}$ & $\begin{array}{l}\text { Weight } \\
(\mathrm{kg})\end{array}$ & $\begin{array}{l}\text { Height } \\
(\mathrm{cm})\end{array}$ & $\begin{array}{c}\text { BMI } \\
\left(\mathrm{kg} / \mathrm{m}^{2}\right)\end{array}$ & $\begin{array}{c}\text { Cervical } \\
\text { dilatation } \\
(\mathrm{cm})\end{array}$ & & & & \\
\hline $\begin{array}{l}\text { Cheng } \\
\text { et al. } \\
{[23]}\end{array}$ & China & $80 / 80$ & $\begin{array}{l}27.5 / \\
27.4\end{array}$ & $39.4 / 39.4$ & NM & NM & $\begin{array}{c}25.9 / \\
26.3\end{array}$ & NM & $\begin{array}{l}\text { When the } \\
\text { cervical } \\
\text { dilation } \\
\text { reached } 3 \mathrm{~cm} \text {, } \\
\text { a catheter was } \\
\text { inserted at the } \\
\text { L3-L4 } \\
\text { interspace, } \\
3-4 \mathrm{~cm} \\
\text { cephaladly. } \\
\text { Initial bolus } \\
13 \mathrm{~mL} \text {, } \\
\text { background } \\
8 \mathrm{~mL} / \mathrm{h} \text {, } \\
\text { lockout } \\
\text { interval } \\
30 \mathrm{~min}\end{array}$ & $\begin{array}{c}\text { PCEA: } \\
0.125 \% \text { or } \\
0.08 \% \\
\text { ropivacaine } \\
\text { with } 0.5 \mu \mathrm{g} / \\
\text { mL DEX; first } \\
\text { dose: } 10 \mathrm{ml} \text {, } \\
\text { background } \\
\text { infusion: } \\
8 \mathrm{ml} / \mathrm{h} \text {, a } \\
\text { single } \\
\text { additional } \\
\text { quantity: } \\
8 \text { ml, and lock } \\
\text { time: } 30 \text { min }\end{array}$ & $\begin{array}{c}\text { PCEA: } \\
0.125 \% \text { or } \\
0.08 \% \\
\text { ropivacaine } \\
\text { with } 0.5 \mu \mathrm{g} / \\
\mathrm{mL} \\
\text { sufentanil; } \\
\text { first dose: } \\
10 \mathrm{ml}, \\
\text { background } \\
\text { infusion: } \\
8 \mathrm{ml} / \mathrm{h}, \mathrm{a} \\
\text { single } \\
\text { additional } \\
\text { quantity: } \\
8 \text { ml, and lock } \\
\text { time } 30 \text { min }\end{array}$ & 7 \\
\hline $\begin{array}{l}\text { Zhang } \\
\text { et al. } \\
{[24]}\end{array}$ & China & $36 / 34$ & $\begin{array}{l}27.3 / \\
26.7\end{array}$ & $39.8 / 40.1$ & $\begin{array}{l}70.8 / \\
69.5\end{array}$ & $\begin{array}{c}159.3 / \\
160.4\end{array}$ & NM & NM & $\begin{array}{l}\text { When the } \\
\text { cervical } \\
\text { dilation } \\
\text { reached } 2 \mathrm{~cm} \text {, } \\
\text { a catheter was } \\
\text { inserted at the } \\
\text { L2-L3 } \\
\text { interspace, } \\
3-4 \mathrm{~cm} \\
\text { cephaladly. } \\
\text { First dose: } \\
10 \mathrm{~mL} \text {, } \\
\text { background: } \\
6 \mathrm{~mL} / \mathrm{h} \text {, } \\
\text { bonus: } 6 \mathrm{~mL} \text {, } \\
\text { lockout time: } \\
20 \mathrm{~min}\end{array}$ & $\begin{array}{c}\text { PCEA: } 0.1 \% \\
\text { ropivacaine } \\
\text { plus } 0.5 \mu \mathrm{g} / \\
\text { mL DEX; first } \\
\text { dose: } 10 \mathrm{ml} \text {, } \\
\text { background } \\
\text { infusion: } \\
6 \mathrm{ml} / \mathrm{h} \text {, a } \\
\text { single } \\
\text { additional } \\
\text { quantity: } \\
6 \text { ml, and lock } \\
\text { time: } 20 \text { min }\end{array}$ & $\begin{array}{c}\text { PCEA: } 0.1 \% \\
\text { ropivacaine } \\
\text { plus } 0.5 \mu \mathrm{g} / \\
\mathrm{mL} \\
\text { sufentanil; } \\
\text { first dose: } \\
10 \mathrm{ml}, \\
\text { background } \\
\text { infusion: } \\
6 \mathrm{ml} / \mathrm{h}, \mathrm{a} \\
\text { single } \\
\text { additional } \\
\text { quantity: } \\
6 \mathrm{ml} \text {, and lock } \\
\text { time: } 20 \mathrm{~min}\end{array}$ & 6 \\
\hline $\begin{array}{l}\text { Li et al. } \\
{[25]}\end{array}$ & China & $\begin{array}{l}291 / \\
287\end{array}$ & $\begin{array}{c}28.8 / \\
29.1\end{array}$ & $39.2 / 39.4$ & $\begin{array}{l}69.4 / \\
68.8\end{array}$ & $\begin{array}{c}160.2 / \\
160.6\end{array}$ & NM & $\mathrm{NM}$ & $\begin{array}{l}\text { When the } \\
\text { cervical } \\
\text { dilation } \\
\text { reached } 2 \mathrm{~cm} \text {, } \\
\text { a catheter was } \\
\text { inserted at the } \\
\text { L2-L3 } \\
\text { interspace, } \\
3-5 \mathrm{~cm} \\
\text { cephaladly. } \\
\text { First dose: } \\
10 \mathrm{~mL} \text {, } \\
\text { background: } \\
6 \mathrm{ml} / \mathrm{h} \text {, bolus: } \\
6 \mathrm{ml} \text { lockout } \\
\text { time: } 15 \mathrm{~min}\end{array}$ & $\begin{array}{c}\text { PCEA: } 0.1 \% \\
\text { ropivacaine } \\
\text { with } 0.5 \mu \mathrm{g} / \\
\text { mL DEX; first } \\
\text { dose: } 10 \mathrm{ml} \text {, } \\
\text { background } \\
\text { infusion: } \\
6 \mathrm{ml} / \mathrm{h}, \mathrm{a} \\
\text { single } \\
\text { additional } \\
\text { quantity: } \\
6 \text { ml, and lock } \\
\text { time: } 15 \text { min }\end{array}$ & $\begin{array}{l}\text { PCEA: } 0.1 \% \\
\text { ropivacaine; } \\
\text { first dose: } \\
10 \mathrm{ml}, \\
\text { background } \\
\text { infusion: } \\
6 \mathrm{ml} / \mathrm{h}, \mathrm{a} \\
\text { single } \\
\text { additional } \\
\text { quantity: } \\
6 \mathrm{ml} \text {, and lock } \\
\text { time: } 15 \text { min }\end{array}$ & 6 \\
\hline
\end{tabular}


TABLE 1: Continued.

\begin{tabular}{|c|c|c|c|c|c|c|c|c|c|c|c|c|}
\hline \multirow[b]{2}{*}{$\begin{array}{l}\text { Study } \\
\text { (year) }\end{array}$} & \multirow[b]{2}{*}{ Country } & \multicolumn{7}{|c|}{ DEX/control } & \multirow[b]{2}{*}{$\begin{array}{l}\text { Epidural } \\
\text { analgesia }\end{array}$} & \multirow[b]{2}{*}{$\begin{array}{c}\text { DEX } \\
\text { intervention }\end{array}$} & \multirow[b]{2}{*}{$\begin{array}{c}\text { Control } \\
\text { intervention }\end{array}$} & \multirow[b]{2}{*}{$\begin{array}{r}\text { Jadad } \\
\text { score }\end{array}$} \\
\hline & & Cases & $\begin{array}{l}\text { Mean } \\
\text { age } \\
\text { (years) }\end{array}$ & $\begin{array}{l}\text { Gestational } \\
\text { week }\end{array}$ & $\begin{array}{l}\text { Weight } \\
(\mathrm{kg})\end{array}$ & $\begin{array}{l}\text { Height } \\
(\mathrm{cm})\end{array}$ & $\begin{array}{c}\text { BMI } \\
\left(\mathrm{kg} / \mathrm{m}^{2}\right)\end{array}$ & $\begin{array}{l}\text { Cervical } \\
\text { dilatation } \\
(\mathrm{cm})\end{array}$ & & & & \\
\hline $\begin{array}{l}\text { Li et al. } \\
\text { [26] }\end{array}$ & China & $36 / 35$ & $\begin{array}{c}29.2 / \\
30.1\end{array}$ & $39.1 / 37.4$ & $\begin{array}{l}65.2 / \\
64.7\end{array}$ & $\begin{array}{c}159.6 / \\
159.0\end{array}$ & NM & NM & $\begin{array}{l}\text { When the } \\
\text { cervical } \\
\text { dilation } \\
\text { reached } 3 \mathrm{~cm} \text {, } \\
\text { a catheter was } \\
\text { inserted at the } \\
\text { L2-L3 } \\
\text { interspace, } \\
3-4 \mathrm{~cm} \\
\text { cephaladly. } \\
\text { First dose: } \\
10 \mathrm{~mL} \text {, } \\
\text { background: } \\
7 \mathrm{ml} / \mathrm{h} \text {, bolus: } \\
7 \mathrm{ml} \text { lockout } \\
\text { time: } 25 \mathrm{~min}\end{array}$ & $\begin{array}{l}\text { PCEA: } 0.1 \% \\
\text { ropivacaine } \\
\text { with } 0.5 \mu \mathrm{g} / \\
\text { mL DEX; first } \\
\text { dose: } 10 \mathrm{ml} \text {, } \\
\text { background } \\
\text { infusion: } \\
7 \mathrm{ml} / \mathrm{h}, \mathrm{a} \\
\text { single } \\
\text { additional } \\
\text { quantity: } \\
7 \text { ml, and lock } \\
\text { time: } 25 \mathrm{~min}\end{array}$ & $\begin{array}{l}\text { PCEA: } 0.1 \% \\
\text { ropivacaine } \\
\text { with } 0.5 \mu \mathrm{g} / \\
\text { ml sufentanil; } \\
\text { first dose: } \\
10 \mathrm{ml} \text {, } \\
\text { background } \\
\text { infusion: } \\
7 \mathrm{ml} / \mathrm{h}, \text { a } \\
\text { single } \\
\text { additional } \\
\text { quantity: } \\
7 \text { ml, and lock } \\
\text { time: } 25 \mathrm{~min}\end{array}$ & 7 \\
\hline $\begin{array}{l}\text { Liu } \\
\text { et al. } \\
{[27]}\end{array}$ & China & $\begin{array}{c}118 / \\
29\end{array}$ & $\begin{array}{c}27.3 / \\
27\end{array}$ & $39.3 / 40$ & $69.5 / 70$ & $\begin{array}{c}160.5 / \\
160\end{array}$ & NM & $3 / 3$ & $\begin{array}{l}\text { A catheter } \\
\text { was inserted } \\
\text { at the L3-L4 } \\
\text { interspace, } \\
3-4 \mathrm{~cm} \\
\text { cephaladly. } \\
\text { First dose: } \\
13 \mathrm{~mL}\end{array}$ & $\begin{array}{c}13 \mathrm{ml} \\
\text { Ropivacaine } \\
\text { with } 0.3,0.4 \text {, } \\
0.5 \text {, or } 0.6 \mu \mathrm{g} / \\
\text { mL DEX }\end{array}$ & $\begin{array}{l}13 \mathrm{ml} \\
\text { ropivacaine }\end{array}$ & 7 \\
\hline
\end{tabular}

DEX, dexmedetomidine; BMI, body mass index; NM, not mentioned; VAS, visual analog scale.

3.4. Sensitivity Analysis and Subgroup Analysis and TSA. To examine the reliability and conclusiveness of the positive results mentioned above, sensitivity analysis was conducted and we found that the incidence of nausea/vomiting was significantly different regardless of which study was omitted, and no heterogeneities were detected (data not shown). To further confirm the conclusiveness of the incidence of nausea/vomiting, TSA was performed, and the $Z$ curve crossed the conventional boundary, TSA boundary, and RIS (Supplementary Figure 12).

Subgroup analysis of all outcomes was conducted through different controls (placebo or opioids). Compared with placebo (Table 3), a significant difference was detected at a VAS score of $30 \mathrm{~min}$ after block $(P<0.00001)$, but sensitivity analysis could not be conducted because only two studies of 158 participants were enrolled. Compared with opioids (Table 3), significant differences were detected regarding the incidences of maternal bradycardia $\left(P=0.04, I^{2}=0 \%\right)$ and nausea/vomiting $\left(P=0.002, I^{2}=0 \%\right)$ with no heterogeneities. To confirm the reliability and conclusiveness of the results of the subgroup analysis, sensitivity analysis was further carried out, and it was only found that the incidence of nausea/ vomiting always had significant differences regardless of which study was omitted (data not shown).

\section{Discussion}

This meta-analysis was performed to investigate the effectiveness and safety of epidural dexmedetomidine during labor analgesia. In total, five RCTs comparing dexmedetomidine with placebo and four RCTs comparing dexmedetomidine with opioids were enrolled for metaanalysis. Pooled data from our meta-analysis found that compared with placebo and opioids, epidural dexmedetomidine decreased the VAS score, MAP, HR, blood loss, and the incidence of nausea/vomiting, and increased the incidence of maternal bradycardia. However, sensitivity analysis only found that the incidence of nausea/vomiting always had significant differences. Moreover, when compared with placebo, subgroup analysis found that dexmedetomidine relieved labor pain and did not increase the incidences of side effects; however, sensitivity analysis of labor pain could not be conducted for only two studies enrolled. When compared with opioids, subgroup analysis found that dexmedetomidine decreased the incidence of nausea/vomiting, increased the incidence of maternal bradycardia, and had similar effect on labor pain. However, sensitivity analysis only found that the incidence of nausea/vomiting always had significant differences. In addition, both meta-analysis and subgroup analysis found no significant differences in other outcomes. Taken together, this meta-analysis found that epidural dexmedetomidine had the potential to alleviate labor pain and did not increase the incidences of adverse effects compared with placebo, provided similar analgesic effects and decreased the incidence of nausea/vomiting compared with opioids, indicating that dexmedetomidine can replace opioids and be safely used in epidural block during labor analgesia.

Although many meta-analyses have demonstrated that single-shot injection of subarachnoid or epidural dexmedetomidine prolongs the duration of analgesia and decreases 


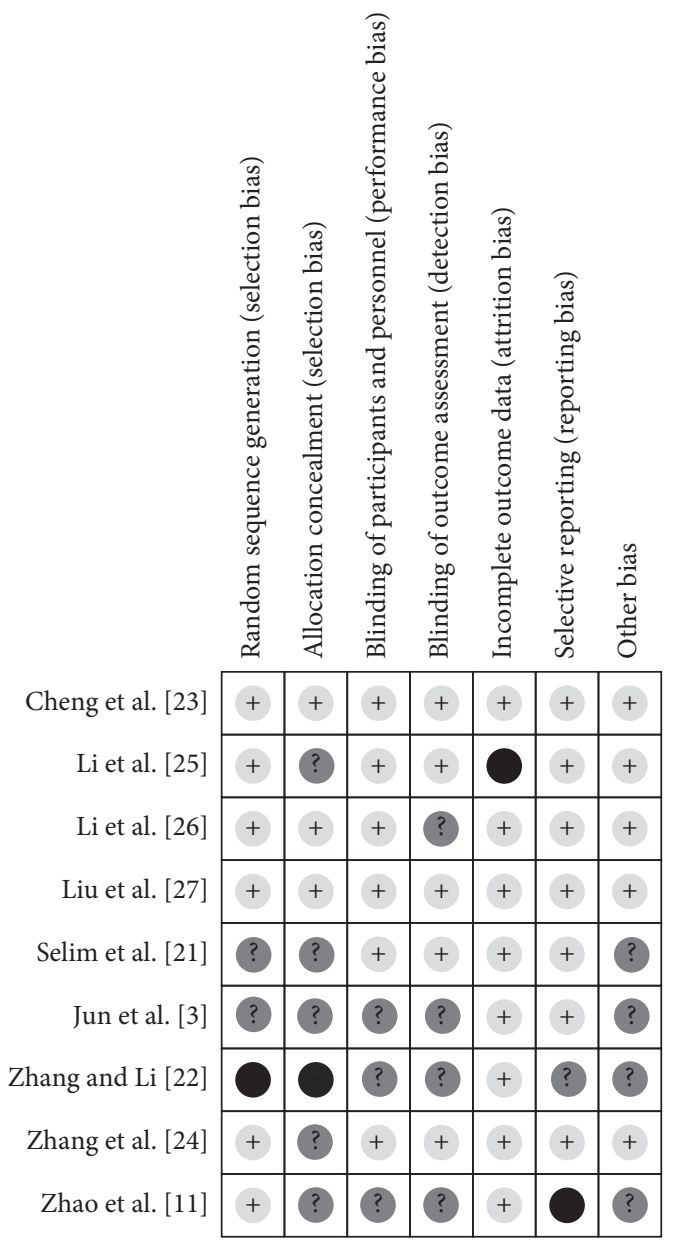

(a)

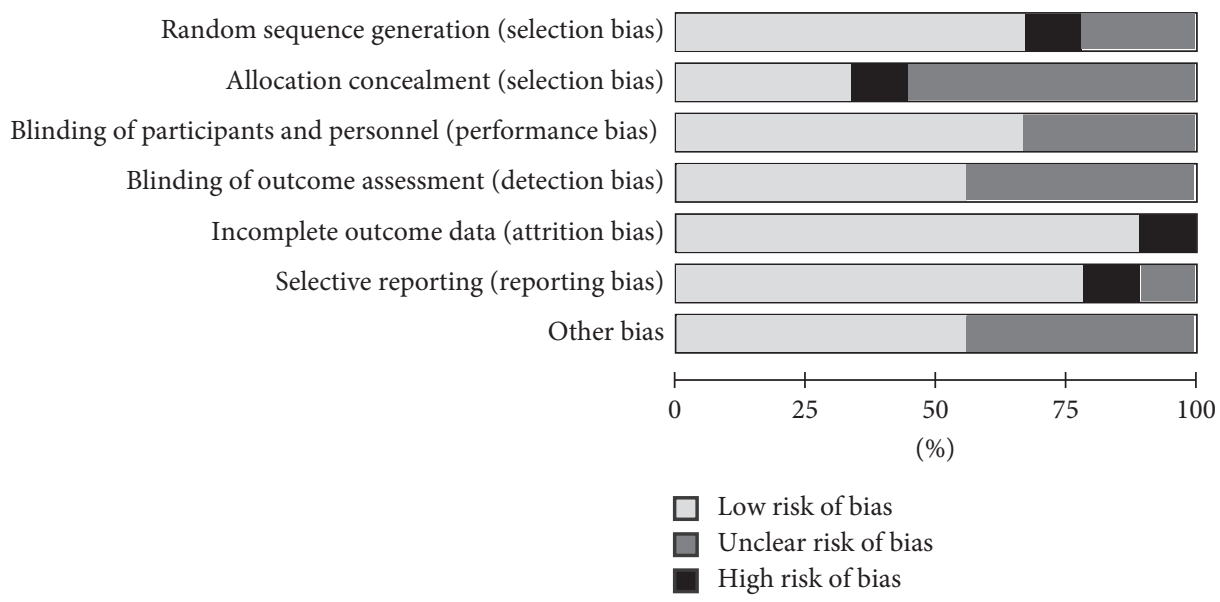

(b)

FIGURE 2: Risk of bias summary (a) and graph (b) of the included studies.

the requirement for rescue analgesia compared with placebo [28-32], opioids [33], or clonidine [34] in different surgical procedures, including cesarean section, no meta-analysis evaluated the effect of epidural dexmedetomidine on labor analgesia. In this meta-analysis, when compared with the control (placebo and opioids), epidural dexmedetomidine relieved labor pain of 30 min after block and the moment of fetal disengagement. However, this difference was unstable by sensitivity analysis. When compared with placebo, dexmedetomidine relieved labor pain of $30 \mathrm{~min}$ after block, but only two studies were enrolled; when compared with opioids, dexmedetomidine showed a similar effect of pain control. Taken together, these results indicate that dexmedetomidine as an adjuvant to local anesthetics may offer 
TABLE 2: Results of meta-analysis.

\begin{tabular}{|c|c|c|c|c|c|}
\hline \multirow{2}{*}{ Outcomes } & \multirow{2}{*}{ Studies } & \multirow{2}{*}{ Participants } & \multicolumn{3}{|c|}{ Effect estimate } \\
\hline & & & $\mathrm{MD}(\mathrm{OR})$ and $95 \% \mathrm{CI}$ & $I^{2}(\%)$ & $P$ \\
\hline VAS score (before block) & 5 & 548 & $-0.02[-0.19,0.16]$ & 15 & 0.85 \\
\hline VAS score ( $15 \mathrm{~min}$ after block) & 2 & 240 & $-2.15[-3.53,-0.77]$ & 92 & 0.002 \\
\hline VAS score (30 min after block) & 4 & 388 & $-0.97[-1.75,-0.19]$ & 93 & 0.01 \\
\hline VAS score (60 min after block) & 3 & 238 & $-1.03[-2.17,0.11]$ & 92 & 0.08 \\
\hline VAS score (120 min after block) & 3 & 232 & $-1.05[-1.90,-0.20]$ & 86 & 0.02 \\
\hline VAS score (at the moment of disengagement) & 3 & 379 & $-0.96[-1.46,-0.46]$ & 94 & 0.0002 \\
\hline Time of first stage & 8 & 1305 & $-18.51[-37.26,0.25]$ & 92 & 0.05 \\
\hline Time of second stage & 8 & 1300 & $1.28[-4.72,7.28]$ & 97 & 0.68 \\
\hline Mode of delivery & 7 & 674 & $1.13[0.70,1.81]$ & 0 & 0.62 \\
\hline Onset of analgesia & 4 & 288 & $0.04[-2.51,2.58]$ & 93 & 0.98 \\
\hline MAP (before block) & 4 & 477 & $0.68[-1.08,2.43]$ & 0 & 0.45 \\
\hline MAP (15 min after block) & 3 & 327 & $-0.93[-8.32,6.46]$ & 91 & 0.81 \\
\hline MAP (30 min after block) & 3 & 317 & $-0.92[-3.78,1.93]$ & 52 & 0.53 \\
\hline MAP (60 min after block) & 2 & 167 & $-3.05[-6.16,0.05]$ & 39 & 0.05 \\
\hline MAP (120 min after block) & 2 & 161 & $-3.74[-6.75,-0.74]$ & 31 & 0.01 \\
\hline MAP (at the moment of disengagement) & 2 & 229 & $0.32[-8.83,9.47]$ & 92 & 0.95 \\
\hline HR (before the block) & 4 & 477 & $-0.41[-1.88,1.05]$ & 0 & 0.58 \\
\hline HR (15 min after block) & 3 & 327 & $-3.33[-10.53,3.87]$ & 93 & 0.36 \\
\hline HR (30 min after block) & 3 & 317 & $-2.70[-4.47,-0.92]$ & 48 & 0.003 \\
\hline HR (60 min after block) & 2 & 167 & $-5.83[-7.78,-3.88]$ & 0 & $<0.00001$ \\
\hline HR (120 min after block) & 2 & 161 & $-4.90[-6.66,-3.13]$ & 0 & $<0.00001$ \\
\hline HR (at the moment of disengagement) & 2 & 229 & $-1.21[-12.73,10.30]$ & 96 & 0.84 \\
\hline Blood loss & 3 & 275 & $-6.03[-10.93,-1.13]$ & 0 & 0.02 \\
\hline Motor block (modified Bromage scale) & 7 & 1162 & $0.29[-0.37,0.96]$ & 95 & 0.39 \\
\hline Apgar score $(1 \mathrm{~min})$ & 4 & 437 & $-0.01[-0.11,0.10]$ & 8 & 0.88 \\
\hline Apgar score ( $5 \mathrm{~min})$ & 5 & 587 & $-0.00[-0.07,0.07]$ & 0 & 0.97 \\
\hline Umbilical artery $\mathrm{pH}$ & 5 & 508 & $0[-0.01,0.02]$ & 0 & 0.72 \\
\hline Umbilical artery $\mathrm{PaO}_{2}$ & 2 & 230 & $1.25[-2.88,5.37]$ & 96 & 0.55 \\
\hline Incidence of maternal bradycardia & 8 & 1323 & $2.90[1.04,8.11]$ & 0 & 0.04 \\
\hline Incidence of hypotension & 7 & 1173 & $1.11[0.38,3.20]$ & 37 & 0.85 \\
\hline Incidence of nausea/vomiting & 9 & 1403 & $0.49[0.30,0.82]$ & 0 & 0.006 \\
\hline Incidence of itching & 6 & 598 & $0.46[0.12,1.81]$ & 21 & 0.27 \\
\hline Incidence of shivering & 3 & 201 & $0.96[0.29,3.16]$ & 0 & 0.95 \\
\hline Incidence of urinary retention & 5 & 511 & $0.49[0.22,1.10]$ & 0 & 0.08 \\
\hline Incidence of fetal heart rate abnormality & 4 & 464 & $0.91[0.40,2.04]$ & 0 & 0.82 \\
\hline
\end{tabular}

MD, mean difference; OR, odds ratio; CI, confidence interval; VAS, visual analog scale; MAP, mean arterial pressure; HR, heart rate.

better pain control during labor than placebo, but more high-quality studies are needed to strengthen this conclusion.

The greatest concern of epidural dexmedetomidine administration is safety. According to these meta-analyses $[28,31]$, although epidural dexmedetomidine significantly reduced the incidence of bradycardia compared with placebo, the incidence of hypotension was not different, and the overall risk of hypotension and bradycardia was statistically insignificant. Similarly, no definite conclusions were reached for epidural dexmedetomidine to increase the incidences of hypotension and bradycardia when compared with placebo and/or opioids in this study. Nausea and vomiting are the most common side effects of epidural anesthesia [28]. Three prior meta-analyses $[28,31,32]$ found that the incidence of nausea/vomiting was not different between epidural dexmedetomidine and placebo. A recent meta-analysis [35] has found that epidural dexmedetomidine significantly reduced the incidence of nausea/vomiting compared with epidural opioids, which is completely consistent with our results.
Taken together, epidural dexmedetomidine has no definite adverse effects on the parturient.

Different from other surgeries, the safety of the fetus should be considered in the cesarean section and labor analgesia. Compared with placebo, spinal anesthesia with intrathecal dexmedetomidine had no significant differences in the 1-min or 5-min Apgar scores or umbilical arterial $\mathrm{pH}$ and $\mathrm{PaO}_{2}$ [29]. In this study, epidural dexmedetomidine had no differences in the duration of stages, mode of delivery, 1min or 5-min Apgar scores, umbilical artery $\mathrm{pH}$ and $\mathrm{PaO}_{2}$, and fetal $\mathrm{HR}$ with placebo and/or opioids, indicating that epidural labor analgesia with dexmedetomidine is safe for fetuses.

Some limitations are present in our meta-analysis. Except for the incidence of nausea/vomiting, due to the small sample size and heterogeneity among the included studies, other outcomes were unable to draw firm conclusions; additional high-quality RCTs are required to fully evaluate these outcomes. The actual dose of dexmedetomidine was not mentioned in all enrolled RCTs; thus, this study did not 
TABLE 3: Subgroup analysis based on different controls (placebo and opioid).

\begin{tabular}{|c|c|c|c|c|c|c|c|c|c|c|}
\hline \multirow{3}{*}{ Outcomes } & \multicolumn{5}{|c|}{ DEX vs. placebo } & \multicolumn{5}{|c|}{ DEX vs. opioids } \\
\hline & \multirow[b]{2}{*}{ Studies } & \multicolumn{4}{|c|}{ Effect estimate } & \multirow[b]{2}{*}{ Studies } & \multirow[b]{2}{*}{ Participants } & \multicolumn{3}{|c|}{ Effect estimate } \\
\hline & & Participants & $\begin{array}{l}\text { MD (OR) and } \\
95 \% \mathrm{CI}\end{array}$ & $\begin{array}{c}I^{2} \\
(\%)\end{array}$ & $P$ & & & $\begin{array}{l}\mathrm{MD}(\mathrm{OR}) \text { and } \\
95 \% \mathrm{CI}\end{array}$ & $\begin{array}{l}I^{2} \\
(\%)\end{array}$ & $P$ \\
\hline VAS score (before block) & 2 & 230 & $\begin{array}{c}0.05[-0.21 \\
0.31]\end{array}$ & 13 & 0.71 & 3 & 318 & $\begin{array}{c}-0.12[-0.40 \\
0.17]\end{array}$ & 17 & 0.41 \\
\hline $\begin{array}{l}\text { VAS score ( } 15 \mathrm{~min} \text { after } \\
\text { block) }\end{array}$ & 1 & 80 & \multicolumn{3}{|c|}{ Not applicable } & 1 & 160 & \multicolumn{3}{|c|}{ Not applicable } \\
\hline $\begin{array}{l}\text { VAS score ( } 30 \mathrm{~min} \text { after } \\
\text { block) }\end{array}$ & 2 & 230 & $\begin{array}{c}-1.56[-1.81 \\
-1.31]\end{array}$ & $30<$ & $<0.00001$ & 2 & 158 & $\begin{array}{c}-0.20[-0.58 \\
0.18]\end{array}$ & 0 & 0.31 \\
\hline $\begin{array}{l}\text { VAS score ( } 60 \mathrm{~min} \text { after } \\
\text { block) }\end{array}$ & 1 & 80 & Not apr & plicable & & 2 & 158 & $\begin{array}{l}-0.64[-1.80 \\
0.52]\end{array}$ & 84 & 0.28 \\
\hline $\begin{array}{l}\text { VAS score (120 min after } \\
\text { block) }\end{array}$ & 1 & 74 & Not app & plicable & & 2 & 158 & $\begin{array}{c}-0.74[-1.65 \\
0.17]\end{array}$ & 79 & 0.11 \\
\hline $\begin{array}{l}\text { VAS score (at the moment of } \\
\text { disengagement) }\end{array}$ & 2 & 219 & $\begin{array}{c}-0.95[-1.87 \\
-0.02]\end{array}$ & 93 & 0.05 & 1 & 160 & \multicolumn{3}{|c|}{ Not applicable } \\
\hline Time of first stage & 5 & 1004 & $\begin{array}{c}-12.44 \\
{[-34.08,9.19]}\end{array}$ & 78 & 0.26 & 3 & 301 & $\begin{array}{c}-27.57 \\
{[-59.46,4.33]}\end{array}$ & 96 & 0.09 \\
\hline Time of second stage & 5 & 999 & $\begin{array}{c}-1.44[-6.52 \\
3.64]\end{array}$ & 92 & 0.58 & 3 & 301 & $\begin{array}{l}5.62[-9.92 \\
21.16]\end{array}$ & 99 & 0.48 \\
\hline Mode of delivery & 3 & 287 & $\begin{array}{c}1.16[0.44 \\
3.05]\end{array}$ & 0 & 0.77 & 4 & 276 & $\begin{array}{l}1.13[0.66 \\
1.93]\end{array}$ & 0 & 0.67 \\
\hline Onset of analgesia & 1 & 60 & \multicolumn{3}{|c|}{ Not applicable } & 3 & 228 & $\begin{array}{c}0.25[-2.89 \\
3.39]\end{array}$ & 95 & 0.87 \\
\hline MAP (before block) & 2 & 230 & $\begin{array}{c}1.89[-0.83 \\
4.60]\end{array}$ & 0 & 0.17 & 2 & 247 & $\begin{array}{c}-0.19[-2.5 \\
2.11]\end{array}$ & 0 & 0.87 \\
\hline MAP (15 min after block) & 1 & 80 & \multicolumn{3}{|c|}{ Not applicable } & 2 & 247 & $\begin{array}{c}1.49[-7.43 \\
10.41]\end{array}$ & 93 & 0.74 \\
\hline MAP (30 min after block) & 2 & 230 & $\begin{array}{c}-1.16[-6.35 \\
4.03]\end{array}$ & 75 & 0.66 & 1 & 87 & \multicolumn{3}{|c|}{ Not applicable } \\
\hline HR (before the block) & 2 & 230 & $\begin{array}{c}-1.33[-3.19 \\
0.54]\end{array}$ & 0 & 0.16 & 2 & 247 & $\begin{array}{c}1.06[-1.31 \\
3.43]\end{array}$ & 0 & 0.38 \\
\hline HR (15 min after block) & 1 & 80 & \multicolumn{3}{|c|}{ Not applicable } & 2 & 247 & $\begin{array}{c}-0.89[-10.80 \\
9.02]\end{array}$ & 95 & 0.86 \\
\hline HR (30 min after block) & 2 & 230 & $\begin{array}{c}-3.14[-6.31 \\
0.04]\end{array}$ & 74 & 0.05 & 1 & 87 & \multicolumn{3}{|c|}{ Not applicable } \\
\hline Blood loss & 2 & 205 & $\begin{array}{c}-6.55[-13.81 \\
0.71]\end{array}$ & 0 & 0.08 & 1 & 70 & \multicolumn{3}{|c|}{ Not applicable } \\
\hline $\begin{array}{l}\text { Motor block (modified } \\
\text { Bromage scale) }\end{array}$ & 3 & 785 & \multicolumn{3}{|c|}{ Not applicable } & 4 & 377 & $\begin{array}{c}0.29[-0.37 \\
0.96]\end{array}$ & 95 & 0.39 \\
\hline Apgar score (1 min) & 2 & 207 & $\begin{array}{c}0.01[-0.08 \\
0.11]\end{array}$ & 0 & 0.79 & 2 & 230 & \multicolumn{3}{|c|}{ Not applicable } \\
\hline Apgar score (5 min) & 3 & 357 & $\begin{array}{c}0.00[-0.07 \\
0.08]\end{array}$ & 0 & 0.89 & 2 & 230 & \multicolumn{3}{|c|}{ Not applicable } \\
\hline Umbilical artery $\mathrm{pH}$ & 2 & 207 & $\begin{array}{l}0.01[-0.01 \\
0.03]\end{array}$ & 0 & 0.35 & 3 & 301 & $\begin{array}{l}0.00[-0.02 \\
0.02]\end{array}$ & 0 & 0.70 \\
\hline Umbilical artery $\mathrm{PaO}_{2}$ & 0 & & \multicolumn{3}{|c|}{ Not applicable } & 2 & 230 & $\begin{array}{c}1.25[-2.88 \\
5.37]\end{array}$ & 96 & 0.55 \\
\hline $\begin{array}{l}\text { Incidence of maternal } \\
\text { bradycardia }\end{array}$ & 4 & 935 & \multicolumn{3}{|c|}{ Not applicable } & 4 & 388 & $\begin{array}{l}2.90[1.04 \\
\quad 8.11]\end{array}$ & 0 & 0.04 \\
\hline Incidence of hypotension & 3 & 785 & $\begin{array}{c}1.59[0.47 \\
5.36]\end{array}$ & 0 & 0.45 & 4 & 388 & $\begin{array}{l}0.68[0.06 \\
\quad 7.98]\end{array}$ & 70 & 0.76 \\
\hline $\begin{array}{l}\text { Incidence of nausea/ } \\
\text { vomiting }\end{array}$ & 5 & 1011 & $\begin{array}{l}0.71[0.37 \\
1.36]\end{array}$ & 0 & 0.30 & 4 & 388 & $\begin{array}{l}0.27[0.12 \\
\quad 0.61]\end{array}$ & 0 & 0.002 \\
\hline Incidence of itching & 2 & 210 & \multicolumn{3}{|c|}{ Not applicable } & 4 & 388 & $\begin{array}{c}0.28[0.07 \\
1.13]\end{array}$ & 2 & 0.07 \\
\hline Incidence of shivering & 1 & 60 & \multicolumn{3}{|c|}{ Not applicable } & 2 & 141 & $\begin{array}{l}0.96[0.26 \\
\quad 3.54]\end{array}$ & 0 & 0.95 \\
\hline $\begin{array}{l}\text { Incidence of urinary } \\
\text { retention }\end{array}$ & 2 & 210 & \multicolumn{3}{|c|}{ Not applicable } & 3 & 301 & $\begin{array}{l}0.49[0.20 \\
\quad 1.15]\end{array}$ & 0 & 0.10 \\
\hline $\begin{array}{l}\text { Incidence of fetal heart rate } \\
\text { abnormality }\end{array}$ & 1 & 147 & \multicolumn{3}{|c|}{ Not applicable } & 3 & 317 & $\begin{array}{l}1.09[0.44 \\
2.70]\end{array}$ & 0 & 0.86 \\
\hline
\end{tabular}

DEX, dexmedetomidine; MD, mean difference; OR, odds ratio; CI, confidence interval; VAS, visual analog scale; MAP, mean arterial pressure; HR, heart rate. 
provide any insight into the effect of different doses of dexmedetomidine. Moreover, eight of the included studies were from China, which resulted in geographical limitations of this study.

\section{Conclusion}

Dexmedetomidine as an adjuvant to epidural local anesthetics has the potential to offer a better analgesic effect than placebo, similar labor pain control to opioids, and has no definite adverse effects on the parturient or fetus. Still, more high-quality RCTs are needed to confirm these conclusions.

\section{Data Availability}

The data used to support the findings of this study are included within the article.

\section{Conflicts of Interest}

The authors declare that they have no conflicts of interest.

\section{Authors' Contributions}

Nijuan Li conceptualized the study, curated the data, and wrote the original draft. Li Hu curated the data and wrote the original draft. Chunping Li was responsible for software and did formal analysis. Xuelin Pan supervised the study and reviewed and edited the manuscript. Yong Tang conceptualized and supervised the study and reviewed and edited the manuscript.

\section{Supplementary Materials}

Supplementary Figure 1: forest plot diagram showing the visual analog scale (VAS) scores. Supplementary Figure 2: forest plot diagram showing the mean arterial pressure (MAP). Supplementary Figure 3: forest plot diagram showing the heart rate (HR). Supplementary Figure 4: forest plot diagram showing the blood loss. Supplementary Figure 5: forest plot diagram showing the incidences of complications. Supplementary Figure 6: forest plot diagram showing the duration of labor stages. Supplementary Figure 7: forest plot diagram showing the mode of delivery. Supplementary Figure 8: forest plot diagram showing the onset of analgesia. Supplementary Figure 9: forest plot diagram showing the level of motor block. Supplementary Figure 10: forest plot diagram showing the Apgar score. Supplementary Figure 11: forest plot diagram showing the umbilical artery $\mathrm{pH}$ and $\mathrm{PaO}_{2}$. Supplementary Figure 12: trial sequential analysis of the incidence of nausea/vomiting compared with placebo and opioids. RIS = required information size. (Supplementary Materials)

\section{References}

[1] M. Van de Velde and B. Carvalho, "Remifentanil for labor analgesia: an evidence-based narrative review," International Journal of Obstetric Anesthesia, vol. 25, pp. 66-74, 2016.

[2] P. Sultan, A. S. Habib, Y. Cho, and B. Carvalho, "The effect of patient warming during caesarean delivery on maternal and neonatal outcomes: a meta-analysis," British Journal of Anaesthesia, vol. 115, no. 4, pp. 500-510, 2015.

[3] W. Jun, A. Zhengzhuang, and O. Li, "Analgesic efficacy of dexmedetomidine used for epidural anesthesia on labor analgesia," Biomedical Research, vol. 29, pp. 426-430, 2018.

[4] C. Wang, S. Liu, C. Han, M. Yu, Y. Hu, and C. Liu, "Effect and placental transfer of dexmedetomidine during caesarean section under epidural anaesthesia," Journal of International Medical Research, vol. 45, no. 3, pp. 964-972, 2017.

[5] S. Parua, D. Choudhury, and M. P. Nath, "Giant haemangioma excision under cervical epidural anaesthesia: a viable alternative to general anaesthesia," Journal of Clinical and Diagnostic Research, vol. 10, pp. UD01-UD02, 2016.

[6] M. Anim-Somuah, R. M. Smyth, and L. Jones, "Epidural versus non-epidural or no analgesia in labour," Cochrane Database of Systematic Reviews, vol. 12, Article ID CD000331, 2011.

[7] S. Mitra, J. Arora, V. Ahuja, and N. Takkar, "Combined spinal-epidural for labor analgesia with low-dose bupivacaine but without any opioid in the spinal component: can we improve upon the traditional?" Acta Anaesthesiologica Belgica, vol. 66, pp. 9-15, 2015.

[8] Y. Nie, Y. Liu, Q. Luo, and S. Huang, "Effect of dexmedetomidine combined with sufentanil for post-caesarean section intravenous analgesia: a randomised, placebo-controlled study," European Journal of Anaesthesiology, vol. 31, no. 4, pp. 197-203, 2014.

[9] J. Mantz, J. Josserand, and S. Hamada, "Dexmedetomidine: new insights," European Journal of Anaesthesiology, vol. 28, no. 1, pp. 3-6, 2011.

[10] W. Zhang and M. Ren, "Optimal dose of epidural dexmedetomidine added to ropivacaine for epidural labor analgesia: a pilot study," Evidence-Based Complementary and Alternative Medicine, vol. 2017, Article ID 7924148, 4 pages, 2017.

[11] Y. Zhao, Y. Xin, Y. Liu, X. Yi, and Y. Liu, "Effect of epidural dexmedetomidine combined with ropivacaine in labor analgesia: a randomized double-blinded controlled study," The Clinical Journal of Pain, vol. 33, no. 4, pp. 319-324, 2017.

[12] M. Cumpston, T. Li, M. J. Page et al., "Updated guidance for trusted systematic reviews: a new edition of the Cochrane handbook for systematic reviews of interventions," Cochrane Database of Systematic Reviews, vol. 10, Article ID ED000142, 2019.

[13] D. Moher, A. Liberati, J. Tetzlaff, and D. G. Altman, "Preferred reporting items for systematic reviews and meta-analyses: the PRISMA statement," International Journal of Surgery, vol. 8, no. 5, pp. 336-341, 2010.

[14] S. Gong, W. Xu, R. Wang et al., "Patient-specific instrumentation improved axial alignment of the femoral component, operative time and perioperative blood loss after total knee arthroplasty," Knee Surgery, Sports Traumatology, Arthroscopy, vol. 27, no. 4, pp. 1083-1095, 2019.

[15] Z.-G. Huang, Y.-H. Feng, Y.-H. Li, and C.-S. Lv, "Systematic review and meta-analysis: Tai Chi for preventing falls in older adults," BMJ Open, vol. 7, no. 2, Article ID e013661, 2017.

[16] S. Li, J. S. W. Kwong, X.-T. Zeng et al., "Plasmakinetic resection technology for the treatment of benign prostatic hyperplasia: evidence from a systematic review and metaanalysis," Scientific Reports, vol. 5, no. 1, Article ID 12002, 2015.

[17] S. Miao, M. Shi, L. Zou, and G. Wang, "Effect of intrathecal dexmedetomidine on preventing shivering in cesarean section after spinal anesthesia: a meta-analysis and trial sequential 
analysis," Drug Design, Development and Therapy, vol. 12, pp. 3775-3783, 2018.

[18] N. Puthenveettil, A. Mohan, S. Rajan, J. Paul, and L. Kumar, "Labor epidural analgesia: comparison of two different intermittent bolus regimes," Anesthesia: Essays and Researches, vol. 12, pp. 832-836, 2018.

[19] T. M. El Menesy and Z. A. El Maaty, "A randomized doubleblind comparative study between low-dose dexmedetomidine and fentanyl in labour epidural analgesia," Egyptian Journal of Anaesthesia, vol. 25, pp. 1-10, 2009.

[20] W. Abdalla, M. Ammar, and A. Tharwat, "Combination of dexmedetomidine and remifentanil for labor analgesia: a double-blinded, randomized, controlled study," Saudi Journal of Anaesthesia, vol. 9, no. 4, pp. 433-438, 2015.

[21] M. F. Selim, A. M. Elnabtity, and A. M. Hasan, "Comparative evaluation of epidural bupivacaine-dexmedetomidine and bupivacaine -fentanyl on Doppler velocimetry of uterine and umbilical arteries during labor," Journal of Prenatal Medicine, vol. 6, pp. 47-54, 2012.

[22] W. Zhang and C. Li, "EC50 of epidural ropivacaine combined with dexmedetomidine for labor analgesia," The Clinical Journal of Pain, vol. 34, no. 10, pp. 950-953, 2018.

[23] Q. Cheng, X. Bi, W. Zhang, Y. Lu, and H. Tian, "Dexmedetomidine versus sufentanil with high- or low-concentration ropivacaine for labor epidural analgesia: a randomized trial," Journal of Obstetrics and Gynaecology Research, vol. 45, no. 11, pp. 2193-2201, 2019.

[24] T. Zhang, Y. Yu, W. Zhang, and J. Zhu, "Comparison of dexmedetomidine and sufentanil as adjuvants to local anesthetic for epidural labor analgesia: a randomized controlled trial," Drug Design, Development and Therapy, vol. 13, pp. 1171-1175, 2019.

[25] L. Li, Z. Yang, and W. Zhang, "Epidural dexmedetomidine for prevention of intrapartum fever during labor analgesia: a randomized controlled trial," Pain and Therapy, vol. 13, pp. 391-400, 2020.

[26] G. Li, Y. Xiao, X. Qi et al., "Combination of sufentanil, dexmedetomidine and ropivacaine to improve epidural labor analgesia effect: a randomized controlled trial," Experimental and Therapeutic Medicine, vol. 20, no. 1, pp. 454-460, 2020.

[27] L. Liu, D. Drzymalski, W. Xu, W. Zhang, L. Wang, and F. Xiao, "Dose dependent reduction in median effective concentration (EC50) of ropivacaine with adjuvant dexmedetomidine in labor epidural analgesia: an up-down sequential allocation study," Journal of Clinical Anesthesia, vol. 68, Article ID 110115, 2020.

[28] X. Zhang, D. Wang, M. Shi, and Y. Luo, "Efficacy and safety of dexmedetomidine as an adjuvant in epidural analgesia and anesthesia: a systematic review and meta-analysis of randomized controlled trials," Clinical Drug Investigation, vol. 37, no. 4, pp. 343-354, 2017.

[29] S. Sun, J. Wang, J. Wang, F. Wang, H. Xia, and S. Yao, "Fetal and maternal responses to dexmedetomidine intrathecal application during cesarean section: a meta-analysis," Medical Science Monitor: International Medical Journal of Experimental and Clinical Research, vol. 26, Article ID e918523, 2020.

[30] X. Liu, X. Zhang, X. Wang, J. Wang, and H. Wang, "Comparative evaluation of intrathecal bupivacaine alone and bupivacaine combined with dexmedetomidine in cesarean section using spinal anesthesia: a meta-analysis," Journal of International Medical Research, vol. 47, no. 7, pp. 2785-2799, 2019.
[31] Y.-Z. Li, Y. Jiang, H. Lin, and X.-P. Yang, "Subarachnoid and epidural dexmedetomidine for the prevention of post-anesthetic shivering: a meta-analysis and systematic review," Drug Design, Development and Therapy, vol. 13, pp. 3785-3798, 2019.

[32] Z. Tu, X. Tan, S. Li, and J. Cui, "The efficacy and safety of dexmedetomidine combined with bupivacaine on caudal epidural block in children: a meta-analysis," Medical Science Monitor, vol. 25, pp. 165-173, 2019.

[33] S. Sun, J. Wang, N. Bao, Y. Chen, and J. Wang, "Comparison of dexmedetomidine and fentanyl as local anesthetic adjuvants in spinal anesthesia: a systematic review and metaanalysis of randomized controlled trials," Drug Design, Development and Therapy, vol. 11, pp. 3413-3424, 2017.

[34] C. Zhang, C. Li, M. Pirrone, L. Sun, and W. Mi, "Comparison of dexmedetomidine and clonidine as adjuvants to local anesthetics for intrathecal anesthesia: a meta-analysis of randomized controlled trials," The Journal of Clinical Pharmacology, vol. 56, no. 7, pp. 827-834, 2016.

[35] M. Qian, F. Gao, J. Liu, and P. Xu, "Dexmedetomidine versus fentanyl as adjuvants to ropivacaine for epidural anaesthesia: a systematic review and meta-analysis," International Journal of Clinical Practice, vol. 75, no. 5, Article ID 13772, 2020. 